\title{
Unraveling the mystery of cancer by secretory microRNA: horizontal microRNA transfer between living cells
}

\section{Nobuyoshi Kosaka and Takahiro Ochiya*}

Division of Molecular and Cellular Medicine, National Cancer Center Research Institute, Tokyo, Japan

Edited by:

Michael Rossbach, Genome Institute of Singapore, Singapore

\section{Reviewed by:}

Lauren Averett Byers, UT MD

Anderson Cancer Center, USA

Leonard Lipovich, Wayne State

University, USA

Raffaele A. Calogero, University of

Torino, Italy

\section{${ }^{*}$ Correspondence:}

Takahiro Ochiya, Division of Molecular and Cellular Medicine, National

Cancer Center Research Institute,

5-1-1, Tsukiji, Chuo-ku, Tokyo

104-0045, Japan.

e-mail: tochiya@ncc.go.jp
microRNAs (miRNAs) have been identified as a fine-tuner in a wide array of biological processes, including development, organogenesis, metabolism, and homeostasis. Deregulation of miRNAs causes diseases, especially cancer. This occurs through a variety of mechanisms, such as genetic alterations, epigenetic regulation, or altered expression of transcription factors, which target miRNAs. Recently, it was discovered that extracellular miRNAs circulate in the blood of both healthy and diseased patients. Since RNase is abundant in the bloodstream, most of the secretory miRNAs are contained in apoptotic bodies, microvesicles, and exosomes or bound to the RNA-binding proteins. However, the secretory mechanism and biological function, as well as the significance of extracellular miRNAs, remain largely unclear. In this article, we summarize the latest and most significant discoveries in recent peer-reviewed research on secretory miRNA involvement in many aspects of physiological and pathological conditions, with a special focus on cancer. In addition, we discuss a new aspect of cancer research that is revealed by the emergence of "secretory miRNA."

Keywords: secretory microRNA, microRNA, exosome, cell-cell communication, cancer

\section{INTRODUCTION}

microRNAs (miRNAs) are small non-coding RNA that repress a wide variety of target genes expression at the post-transcriptional level by sequence-specific base pairing to the $3^{\prime}$ untranslated region of multiple target mRNAs. They are conserved through species, and form an important class of regulators that participate in multiple biological phenomena, including development, organogenesis, and homeostasis. Because of their ability to bind to many target mRNAs (Kwak et al., 2011), once their expression is altered, disease could occur through the deregulation of their target gene networks, particularly that leading to cancer. For this reason, many recent studies have focused on the development of novel diagnosis and therapeutics in the field of oncology. Current studies have revealed that miRNAs are secreted outside of the cells, and their biological significance is beginning to be recognized (Zernecke et al., 2009; Kosaka et al., 2010b; Pegtel et al., 2010). This article is a summary of the latest and most significant findings of original studies on the involvement of secretory miRNAs in cancer, with a special focus on the potential of secretory miRNAs as a humoral factor for cancer biology.

\section{RNA IS NOT ONLY THE MEDIATOR IN THE CENTRAL DOGMA BUT ALSO A SECRETORY FUNCTIONAL MOLECULE}

Before Watson and Crick (1953) described the double-helical structure of the DNA molecule, Mandel and Metais (1947) had found that DNA is present in plasma and serum in 1947. They showed the presence of nucleic acids in healthy subjects as well as in ill patients. After that, many researchers have tried to examine the circulating nucleic acid to develop them as a potential biomarker, especially in the research field of cancer (Fleischhacker and Schmidt, 2007). It is now well documented that RNA can also be detected in plasma, serum, and other body fluids as well as from cell-free supernatants of in vitro cultivated cells. One of the first papers demonstrating the presence of extracellular RNA was published by Stroun et al. (1978). They reported the presence of an RNA form in a nucleoprotein complex spontaneously released from human blood lymphocytes and frog cell systems from auricle cultures. They also showed that the RNA from this complex has a stimulating effect on DNA synthesis in vitro, suggesting the function of secretory RNA in recipient cells.

Meanwhile, the uptake of RNA by recipient cells was also observed. More than 40 years ago, RNAs were reported to be readily taken up by ascites tumor cells (Galand and Ledoux, 1966). In addition, during a study of co-cultured cells that were previously incubated with or without tantalum particles, intact labeled RNA was found to be transferred into the non-labeled recipient cells from labeled donor cells (Kolodny, 1971). Namely, cell-cell communication was mediated not only by proteins, such as cytokines, chemokines, and hormones, but also by secretory RNA.

Given that the concentration of RNA-degrading enzymes, RNase, is high in normal people and even higher in cancer patients (Reddi and Holland, 1976; Tsui et al., 2002) and that RNase is extremely stable, it was reasoned that the RNA released from the cells into the extracellular space must be complexed and in a form that is resistant against RNases. The first study of associating circulating RNA, as RNA-proteolipid complexes, in serum was reported in 1987 (Wieczorek et al., 1987). This study reported a relationship between the presence of RNA-proteolipid complexes and tumor mass/response to therapy. These complexes disappeared $\sim 48 \mathrm{~h}$ after tumor removal and were undetected in benign disorders. Another study demonstrated that the release of a macromolecular substance containing ${ }^{32} \mathrm{P}$ and ${ }^{3} \mathrm{H}$ was found when prelabeled 
Chinese hamster ovary cells were treated with trypsin under conditions in which cells remain fully viable (Rieber and Bacalao, 1974). In contrast, a ribonuclease treatment affected neither the ${ }^{32} \mathrm{P}$ nor the ${ }^{3} \mathrm{H}$ radioactivity. The authors concluded from these experiments that RNA together with glycoproteins is released from the external cell surface.

\section{FUNCTIONAL IMPORTANCE OF SECRETORY mIRNA IN VARIOUS KINDS OF LIFE PHENOMENA}

miRNAs, a class of post-transcriptional gene expression regulators, play critical roles in various kinds of biological phenomena, including development, organogenesis, and homeostasis. Dysregulation of miRNA leads to cancer development and progression and has different expression profiles in normal tissues and cancers (Garzon et al., 2010). For this reason, miRNAs have been investigated for their potential use in the diagnosis, prognosis, and treatment of cancer. miRNAs have recently been detected in human body fluids, including peripheral blood plasma as extracellular nuclease-resistant entities (Kosaka et al., 2010a). Reports in two landmark papers noted that not only mRNAs but also miRNAs were secreted outside of the cells and circulated in human body fluid (Chim et al., 2008; Lawrie et al., 2008). Chim et al. (2008) reported the existence of placental miRNAs in maternal plasma. Interestingly, they showed that the four most abundant placental miRNAs (miR-141, miR-149, miR-299-5p, and miR135b) were detectable in maternal plasma during pregnancy and showed reduced detection rates in post-delivery plasma. Furthermore, Lawrie et al. (2008) investigated whether miRNAs have diagnostic utility by comparing the levels of tumor-associated miR-155, miR-210, and miR-21 in serum from diffuse large Bcell lymphoma patients with healthy controls and showed that the levels were higher in patients than in control sera. These observations support the idea that circulating miRNAs can be used as biomarkers to monitor an individual's health. In addition, these reports also suggest the possibility that secretory miRNA must be contained in or attached to something that could protect RNA from RNase-mediated degradation.

One breakthrough about circulating RNA was the discovery of mRNA and miRNA in exosomes (Valadi et al., 2007). Valadi et al. (2007) showed that mouse and human mast cell-derived exosomes, which are vesicles of endocytic origin released by many kinds of cells that can mediate communication between cells, contain RNA and miRNA. The RNA from mast cell exosomes is transferable to other mouse and human mast cells. After the transfer of mouse exosomal RNA to human mast cells, new mouse proteins were found in the recipient human cells, indicating that transferred exosomal mRNA can be translated after entering another cell. Observations from these three reports indicated one important fact, namely, that miRNA could be existent in the outer space of the cells, where the RNase is present, and could be functional in this new location.

After the discovery of miRNA in exosome, many researchers attempted to identify the function of secretory miRNA because the report from Valadi et al. (2007) had not clarified it in the exosomal miRNA in recipient cells. One of the earliest studies to prove the function of secretory miRNA was revealed by an apoptotic body (Zernecke et al., 2009). They demonstrated that CXCL12 production was mediated by miR-126, which was enriched in apoptotic bodies and repressed the function of the regulator of G protein signaling 16 . This enabled CXCR4 to trigger an autoregulatory feedback loop that increased the production of CXCL12, leading to the recruitment of progenitor cells. This study strongly indicated the importance of a "dying message" for the regulating homeostasis of a healthy status and highlights the functions of miRNAs in health and disease that may extend to the recruitment of progenitor cells during other forms of tissue repair or homeostasis.

After the study of miRNA in apoptotic bodies, three reports showed the function and transfer of secretory miRNAs contained inside the exosome. Pegtel et al. (2010) showed that mature EBVencoded miRNAs are secreted by EBV-infected B cells through exosomes. These EBV-miRNAs repress the EBV target immunoregulatory genes, and these target genes are down-regulated in primary EBV-associated lymphomas. Interestingly, using peripheral blood mononuclear cells from patients with an increased EBV load, these researchers also showed that, although EBV DNA is restricted to the circulating B-cell population, EBV BART miRNAs are present in both B-cell and non-B-cell fractions, suggestive of miRNA transfer in vivo. Zhang et al. (2010) reported that miR-150 is contained inside the exosomes and is secreted from a cultured human monocyte/macrophage cell line and that this exosome delivers miR-150 into human microvascular endothelial cells. Then, elevated exogenous miR-150 effectively reduced c-Myb expression and enhanced cell migration in human microvascular endothelial cells. Our group also demonstrated that a secreted tumor-suppressive miRNA, which is miR-146a down-regulated in prostate cancer, was transported to cancer cells and exerted gene silencing in the recipient prostate cancer cells through the suppression of its target gene, thereby leading to cell growth inhibition (Kosaka et al., 2010b). This suggested that secreted miRNA could function as a cell-cell communication tool between the cancer cells and their microenvironmental cells.

These three reports clarified a variety of physiological and pathological phenomena, including virus infection, vascular disease, and cancer. The variety of research fields highlights the importance of secretory miRNAs in phenomena vital to life. Indeed, recent reports have pointed to various functions of secretory miRNA in many aspects of life, such as cellular communication involving antigen-dependent, unidirectional intercellular transfer of miRNAs by exosomes during immune synapsis (Mittelbrunn et al., 2011), nasopharyngeal carcinoma-mediated transfer of EBV-encoded BART miRNA (Gourzones et al., 2010), hepatocellular carcinoma (Kogure et al., 2011), and cardiovascular diseases (Kuwabara et al., 2011). These reports mainly described the importance of exosomes as an miRNA carrier; however, it is not always the exosome that is important in secretory miRNA-mediated cell-cell communication.

High-density lipoprotein (HDL) transports endogenous miRNAs and delivers them to recipient cells with functional targeting capabilities (Vickers et al., 2011). The human HDL-miRNA profile of normal subjects is significantly different from that of familial hypercholesterolemia subjects. Interestingly, a recent report showed that the mechanism of horizontal transfer of miRNAs is not only dependent on vesicle transfer, such as exosomes, but 
also intercellular connection tools, such as gap junction and RNAbinding protein. Lim et al. (2011) clarified that miRNA was transmitted from bone marrow stroma to breast cancer cells via gap junctions and exosomes in tumor cell quiescence. Arroyo et al. (2011) employed a technique, differential centrifugation and size-exclusion chromatography, to characterize circulating miRNA complexes in human plasma and serum and found that the majority of circulating miRNAs cofractionated with Argonaute2 (Ago2, the key effector protein of miRNA-mediated silencing) protein complexes rather than within vesicles. This study was also confirmed by other groups which have shown Ago2 (Turchinovich et al., 2011) or nucleophosmin 1 as secretory miRNA carriers (Wang et al., 2010). Further biological studies are required to understand the function of miRNAs secreted with an RNAbinding protein, such as Ago 2 or nucleophosmin 1, in a variety of research fields.

To certify the significance of secretory miRNAs in variety of life phenomena, it is also essential to understand the secretion mechanism of miRNA from cells. Previously, we found in HEK293 and COS-7 cells that the secretion of miRNAs was regulated by neutral sphingomyelinase 2 (nSMase 2; Kosaka et al., 2010b), which is the catalytic enzyme of ceramide biosynthesis and is known as an exosome regulatory protein (Trajkovic et al., 2008). The decreased activity of nSMase 2 with a chemical inhibitor, GW4869, and a specific siRNA resulted in the reduced secretion of miRNAs. Complementarily, overexpression of nSMase 2 increased the extracellular amounts of miRNAs. This observation was also confirmed using other cells including T-cells (Mittelbrunn et al., 2011) and hepatocellular carcinoma cells (Kogure et al., 2011). Contrary to our results, inhibition of nSMase 2 significantly increased the amount of miRNAs exported to HDL from macrophages (Vickers et al., 2011).

It remains necessary to elucidate how miRNA is sorted into exosomes or other vesicles, such as microvesicles. Microvesicles, also known as microparticles or shedding vesicles, represent a heterogeneous population of vesicles with a diameter of 100-1000 nm that are released by budding of the plasma (Muralidharan-Chari et al., 2010). It has been shown that microvesicles isolated from embryonic stem cells increase pluripotency of hematopoietic stem cells after horizontal transfer of embryonic stem cell-derived mRNA. Although the functions of microvesicles were recently elucidated, unlike exosome, not only the function but also the sorting mechanisms of miRNAs into microvesicles have not been clarified yet. Furthermore, it has not been shown yet what kind of protein bind to miRNAs in the vesicles such as exosomes, microvesicles, and apoptotic bodies, although Arroyo et al. (2011) clearly showed that circulating Ago2-binding miRNAs were not contained inside vesicles. Gibbings et al. (2009) detected some AGO2 in the purified exosomes, albeit less than in whole-cell lysates, on the contrary, GW182, which required for miRNA function through its binding to AGO2, was dramatically enriched in exosomes. Detecting the proteins, which bind to miRNAs in vesicles, might lead to revealing the sorting mechanism of miRNAs in vesicles. Clarifying the details of the molecular mechanisms of secretory miRNA, such as the manner of cell-cell transfer or secretion mechanisms, will help us understand a variety of diseases, especially cancer (Figure 1).

\section{SECRETORY miRNA AS A HUMORAL FACTOR IN CANCER CELLS}

As shown in this report, secretory miRNAs are functional molecules that modulate many aspects of the biological process. In addition, destruction of the secretion of miRNA from cells might lead to disease, such as cardiovascular diseases, virus infections, deterioration of the immune system, and cancer. From the field of cancer research, we would like to propose two hypotheses regarding secretory miRNA-mediated cancer progression (Figure 2).

One is the function of secretory miRNA in a metastatic niche (Figure 2A). As already shown in several reports, various types of the cells have been shown to have the capability to take up exosomes. The tumor microenvironment is a complex tissue comprising variable numbers of tumor cells, epithelial cells which originated cancer cells, fibroblasts, endothelial cells, and infiltrating leukocytes. Recent reports have explained the mechanism of controlling the cancer cell-mediated phenotypical change of microenvironmental cells through cytokines (Hanahan and Weinberg, 2011). Cytokines are considered as key molecules controlling autocrine or paracrine communications within and between these individual cell types. However, considering the existence of secretory miRNA within these environments, their influence to the cancer niche should be reconsidered. An exosome contains nearly 300 proteins (Atay et al., 2011) with the potential to modulate the state of microenvironment cells. In addition, miRNAs are known to regulate hundreds of target mRNA expressions. Thus, not only exosomal miRNAs but also other types of secretory miRNAs could control the state of cellular phenotypes to the benefit of cancer cells within their niche.

Another hypothesis deals with the function of secretory miRNAs in distant organs (Figure 2B). Recently, Hood et al. (2011) provided evidence of exosome-mediated conditioning of lymph nodes and defined microanatomic responses that enable the metastasis of melanoma cells. Homing of melanoma exosomes to sentinel lymph nodes imposes synchronized molecular signals that affect melanoma cell recruitment, extracellular matrix deposition, and vascular proliferation in the lymph nodes. They showed the physiological importance of exosomes for distal metastasis; however, they have not provided evidence of the molecules species that take part in the modulation of the distal site of metastasis. To reveal the exact function of miRNA targeting sites that are distant from the primary organ, we should identify the molecular mechanisms of the tropism of secretory miRNA transported by carriers.

\section{SECRETORY MIRNA AS A HUMORAL FACTOR IN ORGANISMS}

In this study, systemic transfer of miRNAs has been introduced. However, an active mechanism for the transport of double strand RNA (dsRNA) across tissues and cellular boundaries was found in other organisms, such as nematode and plant. Transmembrane channel-forming protein SID-1 has been shown to mediate passive cellular uptake and cell-to-cell distribution of dsRNA in the nematode C. elegans (Feinberg and Hunter, 2003). In addition, recent report showed that mammalian SID-1 homolog localized to the cell membrane of human cells enhances their uptake of small interfering RNA, resulting in increased siRNA-mediated gene silencing efficacy (Duxbury et al., 2005). Furthermore, although RNA molecules have been implicated in systemic cell-to-cell communication 


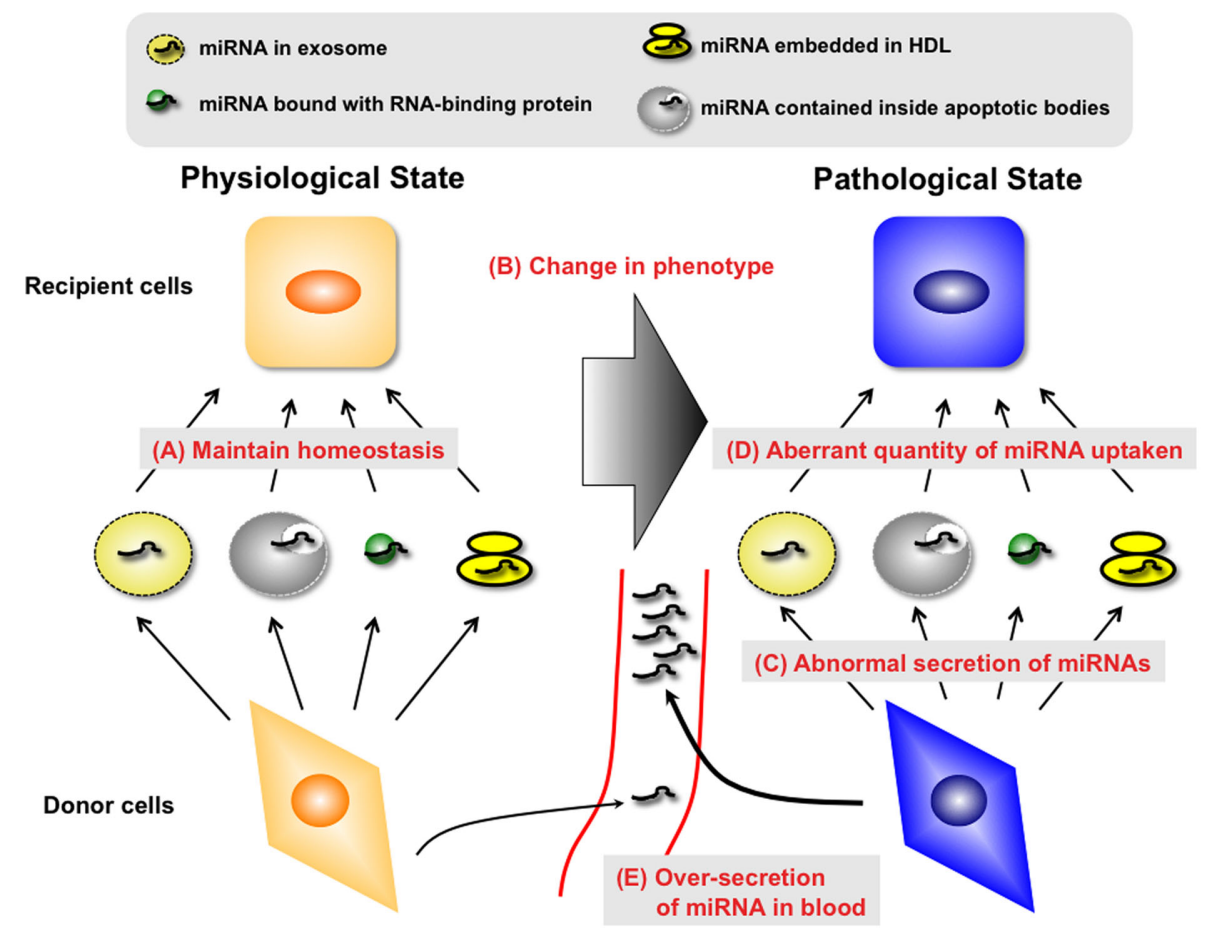

FIGURE 1 | Unveiling the mechanisms and roles of secretory miRNAs in both physiological and pathological conditions is essential for the development of novel diagnostic methods and therapeutics to treat various human diseases. In the physiological condition, secretory miRNAs may maintain the homeostasis of organs and tissues via diverse types of miRNA carriers (A). However, this state may be destroyed by the change of phenotype in donor cells or recipient cells (B). From the viewpoint of donor cells, changes in the phenotype of donor cells may lead to the alteration of
miRNA species with carriers (C). Furthermore, the quantity of secretory miRNA uptake by recipient cells may change in this situation (D). It is necessary to understand these abnormal changes of molecular mechanisms for the development of a therapy against "secretory miRNAs-mediated diseases." In addition, it is possible that the amount of secretory miRNAs changes in this situation through the abnormal regulation of carrier production (E). Understanding these changes may lead to the exploitation of a diagnosis marker for "secretory miRNAs-mediated diseases." in plants (Chitwood and Timmermans, 2010), recent studies have shown that miRNAs are mobile signals that control gene expression during plant development (Dunoyer et al., 2010; Molnar et al., 2010), suggesting that the transfer of RNA is found globally in organisms.

Surprisingly, Zhang et al. (2011) reported that exogenous plant miRNAs are present in the sera and tissues of various animals and that these exogenous plant miRNAs are primarily acquired orally, through food intake. Rice abundant miRNA, miR-168a, is one of the most highly enriched exogenous plant miRNAs in the sera of Chinese subjects. Furthermore, they also found that MIR168a could bind to the human/mouse low-density lipoprotein receptor adapter protein 1 (LDLRAP1) mRNA, inhibit LDLRAP1 expression in liver, and consequently decrease LDL removal from mouse plasma. This study prompted the idea that miRNAs could regulate the gene expression across the kingdom. In addition, one of the important point of this study is that identification of plant miRNAs in human peripheral blood was performed by Solexa sequencing. High-throughput transcriptome analysis by Next Generation Sequencing, specifically RNAseq, is currently widely available. As shown in the case of rice miRNAs, these techniques may help answer the question about the novel small RNAs recently discovered to be secreted.

\section{FUTURE DIRECTIONS FOR RESEARCH ON SECRETORY RNAs}

In this review, we summarized the recent findings of secretory miRNAs. The research field of secretory miRNAs has just begun. To use the knowledge of secretory miRNAs for human health, we should unveil the mystery of secretory RNA as follows.

First, we need to know the all kinds of secretory RNA species. Interestingly, Dinger et al. (2008) independently analyzed the microarray dataset from Valadi et al.'s (2007) study and found that many longer non-coding RNAs (ncRNAs) were also present in exosomes, including a number of ncRNAs associated with important genes and several known ncRNAs, such as Copg2as and Nespas, in mast cell-derived exosomes. This question seems quite easy to obtain the answer. As we already mentioned above, recent development of next generation sequencing technologies has been developed. This means that we can directly recognize the nucleic acids that can exist outer space of the cells.

Second, secretory machinery of miRNAs and other types of RNA should be clarified. As described in this paper, we recently detected the part of miRNAs secretion mechanism mediated by exosome (Kosaka et al., 2010b). Analyzing the secretion mechanism of various kinds of RNAs and sorting mechanism of miRNA into the vesicles leads the development of novel nucleic acids based medicine. 
A
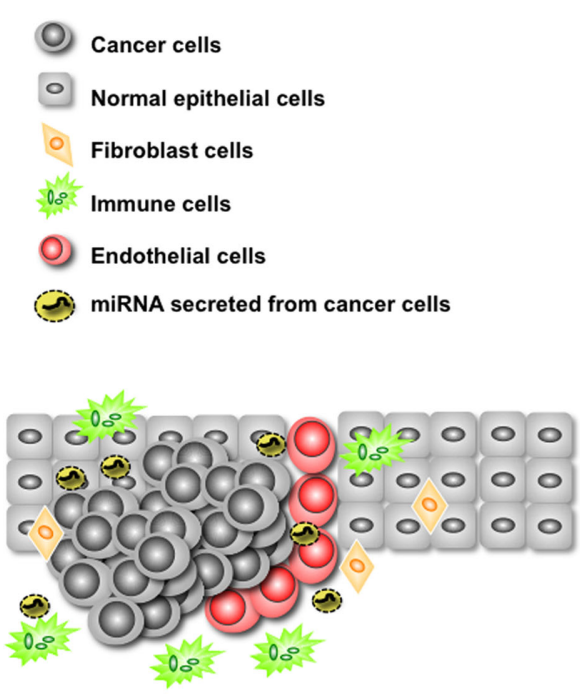

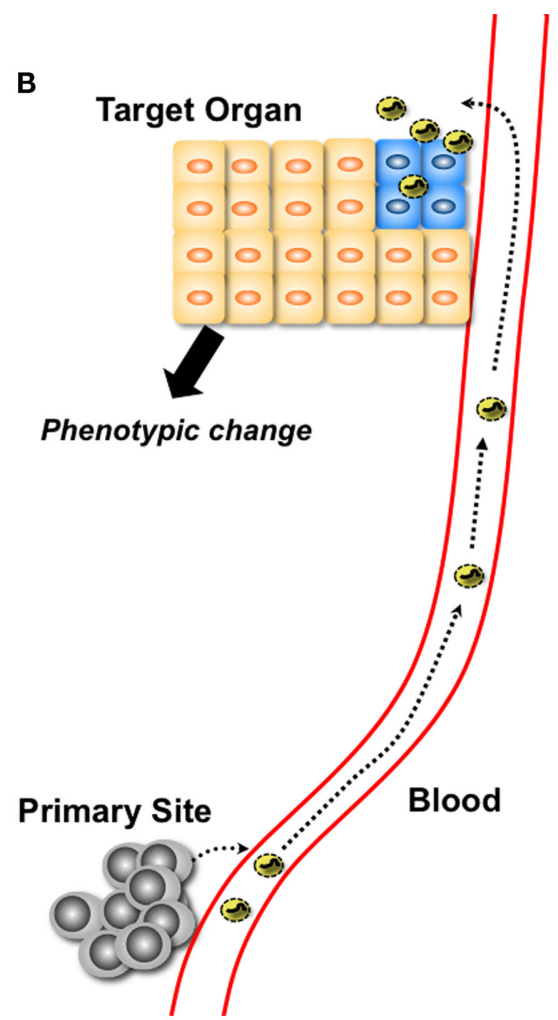

cancer cells control the behavior of microenvironmental cells for their own benefit. (B) Secretory miRNAs could be functional molecules in a distant organ.
miRNA-mediated cancer progression. (A) Secretory miRNAs mediate the modulation of microenvironmental cells. Secretory miRNAs from
Last point is to know the function of secretory miRNAs in more detail, such as physiological conditions and pathological conditions. Reports on the function of secretory miRNAs in physiological conditions, such as embryogenesis, organogenesis, and maintaining tissue and organ homeostasis, are not available. In addition, to know the function of secretory miRNAs, we need to know the incorporation mechanism of miRNAs. Although SID-1 found in C. elegans is good example of secretory miRNA transport mechanism, the other machinery might exist in vertebrates. Indeed, both transporter-dependent (SID-1 dependent) and transporter-independent (SID-1 independent) dsRNA export takes place from C. elegans cells (Jose et al., 2009). Furthermore, as shown previously, miRNA from plant was detected in human circulating peripheral blood, and the function of plant derived miRNA was documented by the authors, suggesting that other types of small RNA from other species might contribute to the regulating of physiological or pathological situation. Because miRNAs act as multi-functional molecules via the binding to sequence similarities and regulate various life phenomena, secretory miRNAs

\section{REFERENCES}

Arroyo, J. D., Chevillet, J. R., Kroh, E. M., Ruf, I. K., Pritchard, C. C., Gibson, D. F., Mitchell, P. S., Bennett, C. F., Pogosova-Agadjanyan, E. L., Stirewalt, D. L., Tait, J. F., and Tewari, M. (2011). Argonaute2 complexes carry a population of circulating microRNAs independent of vesicles in human plasma. Proc. Natl. Acad. Sci. U.S.A. 108, 5003-5008.

Atay, S., Gercel-Taylor, C., Kesimer, M., and Taylor, D. D. (2011).

might be a humoral factor that exerts its influence in distant organs, similarly to hormones. Clarifying the species, mechanisms and roles of secretory miRNA, and other secretory ncRNAs in both pathological and physiological conditions would unveil the mystery of "secretory miRNAs-mediated disease" (Figure 1).

\section{ACKNOWLEDGMENTS}

This work was supported in part by a grant-in-aid for the ThirdTerm Comprehensive 10-Year Strategy for Cancer Control, a grantin-aid for Scientific Research on Priority Areas Cancer from the Ministry of Education, Culture, Sports, Science, and Technology, the Program for Promotion of Fundamental Studies in Health Sciences of the National Institute of Biomedical Innovation, and the Japan Society for the Promotion of Science through the "Funding Program for World-Leading Innovative R\&D on Science and Technology (FIRST Program)" initiated by the Council for Science and Technology Policy. We apologize to colleagues whose work we could not cite owing to space limitations. We are grateful for Dr. Nami Nogawa-Kosaka for critical reading of the manuscript.

Morphologic and proteomic characterization of exosomes released by cultured extravillous trophoblast cells. Exp. Cell Res. 317, 1192-1202.

Chim, S. S., Shing, T. K., Hung, E. C., Leung, T. Y., Lau, T. K., Chiu, R.
W., and Lo, Y. M. (2008). Detection and characterization of placental microRNAs in maternal plasma. Clin. Chem. 54, 482-490.

Chitwood, D. H., and Timmermans, M. C. (2010). Small RNAs are on the move. Nature 467, 415-419. 
Dinger, M. E., Mercer, T. R., and Mattick, J. S. (2008). RNAs as extracellular signaling molecules. J. Mol. Endocrinol. 40, 151-159.

Dunoyer, P., Schott, G., Himber, C., Meyer, D., Takeda, A., Carrington, J. C., and Voinnet, O. (2010). Small RNA duplexes function as mobile silencing signals between plant cells. Science 328, 912-916.

Duxbury, M. S., Ashley, S. W., and Whang, E. E. (2005). RNA interference: a mammalian SID-1 homologue enhances siRNA uptake and gene silencing efficacy in human cells. Biochem. Biophys. Res. Commun. 3, 459-463.

Feinberg, E. H., and Hunter, C. P. (2003). Transport of dsRNA into cells by the transmembrane protein SID-1. Science 12, 1545-1547.

Fleischhacker, M., and Schmidt, B. (2007). Circulating nucleic acids (CNAs) and cancer - a survey. Biochim. Biophys. Acta 1775, 181-232.

Galand, P., and Ledoux, L. (1966). Uptake of exogenous ribonucleic acid by ascites tumor cells. II. Relations between RNA uptake and the cellular metabolism. Exp. Cell Res. 43, 391-397.

Garzon, R., Marcucci, G., and Croce, C. M. (2010). Targeting microRNAs in cancer: rationale, strategies and challenges. Nat. Rev. Drug Discov. 9, 775-789.

Gibbings, D. J., Ciaudo, C., Erhardt, M., and Voinnet, O. (2009). Multivesicular bodies associate with components of miRNA effector complexes and modulate miRNA activity. Nat. Cell Biol. 11, 1143-1149.

Gourzones, C., Gelin, A., Bombik, I., Klibi, J., Verillaud, B., Guigay, J., Lang, P., Temam, S., Schneider, V., Amiel, C., Baconnais, S., Jimenez, A. S., and Busson, P. (2010). Extracellular release and blood diffusion of BART viral micro-RNAs produced by EBV-infected nasopharyngeal carcinoma cells. Virol. J. 7, 271.

Hanahan, D., and Weinberg, R. A. (2011). Hallmarks of cancer: the next generation. Cell 144, 646-674.

Hood, J. L., San, R. S., and Wickline, S. A. (2011). Exosomes released by melanoma cells prepare sentinel lymph nodes for tumor metastasis. Cancer Res. 71, 3792-3801.

Jose, A. M., Smith, J. J., and Hunter, C. P. (2009). Export of RNA silencing from $C$. elegans tissues does not require the RNA channel SID1. Proc. Natl. Acad. Sci. U.S.A. 106, 2283-2288.

Kogure, T., Lin, W. L., Yan, I. K., Braconi, C., and Patel, T. (2011). Intercellular nanovesicle-mediated microRNA transfer: a mechanism of environmental modulation of hepatocellular cancer cell growth. Hepatology 54, 1237-1248.

Kolodny, G. M. (1971). Evidence for transfer of macromolecular RNA between mammalian cells in culture. Exp. Cell Res. 65, 313-324.

Kosaka, N., Iguchi, H., and Ochiya, T. (2010a). Circulating microRNA in body fluid: a new potential biomarker for cancer diagnosis and prognosis. Cancer Sci. 101, 2087-2092.

Kosaka, N., Iguchi, H., Yoshioka, Y., Takeshita, F., Matsuki, Y., and Ochiya, T. (2010b). Secretory mechanisms and intercellular transfer of microRNAs in living cells. J. Biol. Chem. 285, 17442-17452.

Kuwabara, Y., Ono, K., Horie, T., Nishi, H., Nagao, K., Kinoshita, M., Watanabe, S., Baba, O., Kojima, Y., Shizuta, S., Imai, M., Tamura, T., Kita, T. and Kimura, T. (2011). Increased microRNA-1 and microRNA-133a levels in serum of patients with cardiovascular disease indicate the existence of myocardial damage. Circ. Cardiovasc. Genet. 4, 446-454.

Kwak, P. B., Iwasaki, S., and Tomari, Y. (2011). The microRNA pathway and cancer. Cancer Sci. 101, 2309-2315.

Lawrie, C. H., Gal, S., Dunlop, H. M. Pushkaran, B., Liggins, A. P., Pulford, K., Banham, A. H., Pezzella, F., Boultwood, J., Wainscoat, J. S., Hatton, C. S., and Harris, A. L. (2008). Detection of elevated levels of tumour-associated microRNAs in serum of patients with diffuse large B-cell lymphoma. Br. J. Haematol. 141, 672-675.

Lim, P. K., Bliss, S. A., Patel, S. A., Taborga, M., Dave, M. A., Gregory, L. A., Greco, S. J., Bryan, M., Patel, P. S., and Rameshwar, P. (2011). Gap junction-mediated import of microRNA from bone marrow stromal cells can elicit cell cycle quiescence in breast cancer cells. Cancer Res. 71, 1550-1560.

Mandel, P., and Metais, P. (1947). Les acides nucleiques du plasma sanguin chez l'homme. C. R. Acad. Sci. Paris 142, 241-243.

Mittelbrunn, M., Gutierrez-Vazquez, C., Villarroya-Beltri, C., Gonzalez, S., Sanchez-Cabo, F., Gonzalez, M. A., Bernad, A., and Sanchez-Madrid, F. (2011). Unidirectional transfer of microRNA-loaded exosomes from $\mathrm{T}$ cells to antigen-presenting cells. Nat. Commun. 2, 282.

Molnar, A., Melnyk, C. W., Bassett, A., Hardcastle, T. J., Dunn, R., and Baulcombe, D. C. (2010). Small silencing RNAs in plants are mobile and direct epigenetic modification in recipient cells. Science 328, 872-875.
Muralidharan-Chari, V., Clancy, J. W., Sedgwick, A., and D'Souza-Schorey, C. (2010). Microvesicles: mediators of extracellular communication during cancer progression. J. Cell. Sci. $15,1603-1611$.

Pegtel, D. M., Cosmopoulos, K. Thorley-Lawson, D. A., Van Eijndhoven, M. A., Hopmans, E. S., Lindenberg, J. L., De Gruijl, T. D., Wurdinger, T., and Middeldorp, J. M. (2010). Functional delivery of viral miRNAs via exosomes. Proc. Natl. Acad. Sci. U.S.A. 107, 6328-6333.

Reddi, K. K., and Holland, J. F. (1976). Elevated serum ribonuclease in patients with pancreatic cancer. Proc. Natl. Acad. Sci. U.S.A. 73, 2308-2310.

Rieber, M., and Bacalao, J. (1974). An "external” RNA removable from mammalian cells by mild proteolysis. Proc. Natl. Acad. Sci. U.S.A. 71 , 4960-4964.

Stroun, M., Anker, P., Beljanski, M. Henri, J., Lederrey, C., Ojha, M., and Maurice, P. A. (1978). Presence of RNA in the nucleoprotein complex spontaneously released by human lymphocytes and frog auricles in culture. Cancer Res. 38, 3546-3554.

Trajkovic, K., Hsu, C., Chiantia, S., Rajendran, L., Wenzel, D., Wieland, F., Schwille, P., Brugger, B., and Simons, M. (2008). Ceramide triggers budding of exosome vesicles into multivesicular endosomes. Science 319, 1244-1247.

Tsui, N. B., Ng, E. K., and Lo, Y. M. (2002). Stability of endogenous and added RNA in blood specimens, serum, and plasma. Clin. Chem. 48, 1647-1653.

Turchinovich, A., Weiz, L., Langheinz, A., and Burwinkel, B. (2011). Characterization of extracellular circulating microRNA. Nucleic Acids Res. 39, 7223-7233.

Valadi, H., Ekstrom, K., Bossios, A., Sjostrand, M., Lee, J. J., and Lotvall, J. O. (2007). Exosome-mediated transfer of mRNAs and microRNAs is a novel mechanism of genetic exchange between cells. Nat. Cell Biol. 9, 654-659.

Vickers, K. C., Palmisano, B. T., Shoucri, B. M., Shamburek, R. D., and Remaley, A. T. (2011). MicroRNAs are transported in plasma and delivered to recipient cells by highdensity lipoproteins. Nat. Cell Biol. 13, 423-433.

Wang, K., Zhang, S., Weber, J., Baxter, D., and Galas, D. J. (2010). Export of microRNAs and microRNA-protective protein by mammalian cells. Nucleic Acids Res. 38, 7248-7259.

Watson, J. D., and Crick, F. H. (1953). Molecular structure of nucleic acids; a structure for deoxyribose nucleic acid. Nature 171, 737-738.

Wieczorek, A. J., Sitaramam, V., Machleidt, W., Rhyner, K., Perruchoud, A. P., and Block, L. H. (1987). Diagnostic and prognostic value of RNAproteolipid in sera of patients with malignant disorders following therapy: first clinical evaluation of a novel tumor marker. Cancer Res. 47, 6407-6412.

Zernecke, A., Bidzhekov, K., Noels, H., Shagdarsuren, E., Gan, L., Denecke, B., Hristov, M., Koppel, T., Jahantigh, M. N., Lutgens, E., Wang, S., Olson, E. N., Schober, A., and Weber, C. (2009). Delivery of microRNA126 by apoptotic bodies induces CXCL12-dependent vascular protection. Sci. Signal. 2, ra81.

Zhang, L., Hou, D., Chen, X., Li, D., Zhu, L., Zhang, Y., Li, J., Bian, Z., Liang, X., Cai, X., Yin, Y., Wang, C., Zhang, T., Zhu, D., Zhang, D., Xu, J., Chen, Q., Ba, Y., Liu, J., Wang, Q., Chen, J., Wang, J., Wang, M., Zhang, Q., Zhang, J., Zen, K., and Zhang, C.Y. (2011). Exogenous plant MIR168a specifically targets mammalian LDLRAP1: evidence of crosskingdom regulation by microRNA. Cell Res. PMID: 21931358. [Epub ahead of print]

Zhang, Y., Liu, D., Chen, X., Li, J., Li, L., Bian, Z., Sun, F., Lu, J., Yin, Y., Cai, X., Sun, Q., Wang, K., Ba, Y., Wang, Q., Wang, D., Yang, J., Liu, P., Xu, T., Yan, Q., Zhang, J., Zen, K., and Zhang, C. Y. (2010). Secreted monocytic miR-150 enhances targeted endothelial cell migration. Mol. Cell 39, 133-144.

Conflict of Interest Statement: The authors declare that the research was conducted in the absence of any commercial or financial relationships that could be construed as a potential conflict of interest.

Received: 29 August 2011; paper pending published: 20 September 2011; accepted: 08 December 2011; published online: 03 January 2012.

Citation: Kosaka N and Ochiya T (2012) Unraveling the mystery of cancer by secretory microRNA: horizontal microRNA transfer between living cells. Front. Gene. 2:97. doi: 10.3389/fgene.2011.00097

This article was submitted to Frontiers in Non-Coding RNA, a specialty of Frontiers in Genetics.

Copyright (c) 2012 Kosaka and Ochiya. This is an open-access article distributed under the terms of the Creative Commons Attribution Non Commercial License, which permits non-commercial use, distribution, and reproduction in other forums, provided the original authors and source are credited. 